\title{
The PCDH1 gene and asthma in early childhood
}

\author{
Li J. Mortensen ${ }^{1}$, Eskil Kreiner-Møller ${ }^{1}$, Hakon Hakonarson², Klaus Bønnelykke \\ and Hans Bisgaard ${ }^{1}$
}

Affiliations: 'Copenhagen Prospective Studies on Asthma in Childhood, Danish Pediatric Asthma Center, Health Sciences, University of Copenhagen, Copenhagen University Hospital, Gentofte, Denmark. ${ }^{2}$ Center for Applied Genomics, Dept of Pediatrics and Division of Human Genetics, Children Hospital of Philadelphia, University of Pennsylvania, Pennsylvania, PA, USA.

Correspondence: H. Bisgaard, Copenhagen Prospective Studies on Asthma in Childhood, Danish Pediatric Asthma Center, Health Sciences, University of Copenhagen, Copenhagen University Hospital, Gentofte, Ledreborg Allé 34, 2820 Gentofte, Denmark. E-mail: bisgaarddacopsac.com

ABSTRACT Previous studies have suggested that variants in the protocadherin-1 (PCDH1) gene, which is important for cell-cell adhesion, are associated with asthma, bronchial, hyperresponsiveness and atopic dermatitis in school children.

Our aim was to associate common variants of the $P C D H 1$ gene with longitudinally assessed asthma phenotypes and atopic dermatitis in early childhood. We analysed eight single-nucleotide polymorphisms in PCDH1 from 411 children born to asthmatic mothers from the Copenhagen Prospective Studies on Asthma in Childhood birth cohort. Asthma and atopic dermatitis were diagnosed prospectively to the age of 7 years and asthma was categorised by temporal pattern: transient early respiratory symptoms, persistent symptoms and late-onset symptoms. Bronchial responsiveness was measured at age 6 years. We used additive genetic models.

Kaplan-Meier plots revealed early onset in hetero- and homozygotes for the rs10063472-T allele. Significant association was observed between the transient early phenotype and rs10063472-T (transient early versus all: OR 1.91, 95\% CI 1.21-3.01, $\mathrm{p}=0.0058$; transient early versus asymptomatic: OR 2.00, 95\% CI $1.23-3.25, \mathrm{p}=0.0053)$. No association was observed for other symptom patterns or bronchial responsiveness. Significant association was observed for atopic dermatitis and rs11167761-A (OR 1.85, 95\% CI 1.24-2.75, $\mathrm{p}=0.0026)$.

Common variations in $\mathrm{PCDH} 1$ increase the risk of developing both transient early asthma and atopic dermatitis in early childhood.

@ERSpublications

Common variations in the $P C D H 1$ gene increase the risk of developing both asthma and atopic dermatitis in early life http://ow.ly/sjbNL

For editorial comments see page 671.

This article has supplementary material available from www.erj.ersjournals.com

Received: Feb 042013 | Accepted after revision: July 162013 | First published online: Aug 292013

Support statement: Copenhagen Prospective Studies on Asthma in Childhood (COPSAC) is funded by private and public research funds. The Lundbeck Foundation, the Pharmacy Foundation of 1991, Augustinus Foundation, Danish Lung Association, the Danish Medical Research Council and The Danish Paediatric Asthma Centre provided core support for COPSAC. A full list of donors is available at www.copsac.com/content/copsac-fully-externally-funded The funding agencies did not have any role in study design, data collection and analysis, decision to publish, or preparation of the manuscript.

Conflict of interest: Disclosures can be found alongside the online version of the article at www.erj.ersjournals.com

Copyright @ERS 2014 


\section{Introduction}

Asthma is the most common chronic disease in children and it is a major cause of emergency department visits, hospitalisations and school absence $[1,2]$. The incidence of childhood asthma seems to be increasing despite improved treatments over the last few decades [1]. The development of asthma is a complex interaction between environmental and genetics factors [3]. It is defined as a chronic inflammatory disorder with bronchial hyperresponsiveness (BHR) that leads to recurrent episodes of wheezing, breathlessness, chest tightness and coughing, particularly at night or in the early morning, and it is usually associated with widespread, but variable, airflow obstruction within the lungs, which is reversible either spontaneously or with treatment [4]. The different respiratory phenotypic subtypes in early childhood, especially under the age of 3 years, vary significantly over time and are associated with different risk factors [5,6]. New insight into the disease pathogenesis and the finding of new genes might improve management through improved understanding of subphenotypes.

Several genes are reported to have an effect on the development of asthma [7]. Previous research has shown that polymorphisms in the gene encoding protocadherin-1 (PCDH1) are associated with BHR, asthma among adults and school children, and the development of atopic dermatitis among children $[8,9]$. Interactions with in utero exposition to tobacco smoke have been proposed to affect development of BHR in children [8]. PCDH1 belongs to a large group of cell-cell adhesion molecules. It is expressed in several tissues, including airway epithelium and skin keratinocytes [10, 11]. It shows intracellular signalling abilities, is thought to play a role in cell repair and is expressed during fetal lung development in mice [12-15]. The PCDH1 gene is $25 \mathrm{~kb}$ long, and is positioned on chromosome 5q31-q33. It consists of five exons and, through alternative splicing, two annotated protein isoforms [16].

Children with asthmatic symptoms are at risk of developing atopic dermatitis and the diseases are, therefore, believed to share some common background, both genetically and environmentally [17]. It is plausible that genetic variations altering epithelial cell repair and/or epithelial barrier function cause development of both asthma and atopic dermatitis.

The aim of this study was to associate common variants of the PCDH1 gene with different asthma phenotypes and atopic dermatitis in young children followed since birth.

\section{Method}

Study population

The Copenhagen Prospective Studies on Asthma in Childhood 2000 study (COPSAC2000) is a single-centre, prospective clinical birth-cohort study of 411 children born to asthmatic mothers, with the objective of investigating the gene-environment phenotype relationship in the development of atopic diseases [18]. The study was approved by the Copenhagen Ethics Committee (KF 01-289/96) and the Danish Data Protection Agency (2008-41-1754).

\section{Clinical assessment and diagnosing \\ Recurrent episodes of troublesome lung symptoms}

Troublesome lung symptoms (TLS) were defined as breathlessness, wheeze and recurrent cough severely affecting sleep, activity and/or the well being of the child. "Recurrent TLS" were defined as: three consecutive days of TLS, five times within 6 months; TLS for four consecutive weeks; or one episode of severe asthmatic symptoms requiring hospitalisation [18].

\section{Recurrent TLS phenotypes}

Children with recurrent TLS were categorised into three groups by symptom pattern [19], as follows. 1) Transient early respiratory symptoms: debut of symptoms and symptom remission within the first 3 years of life. 2) Late-onset respiratory symptoms: debut of symptoms after the age of 3 years. 3) Persistent respiratory symptoms: debut of symptoms before the age of 3 years, continuing after the age of 3 years.

\section{Asthma diagnosis}

Doctors at the research unit diagnosed asthma according to a predefined algorithm based on recurrent TLS and relapse after discontinued inhaled corticosteroid (ICS) treatment. The algorithm has been previously described in detail [18].

Atopic dermatitis

Skin rash on the child was evaluated according to Hanifin and Rajka criteria [18, 20]. 
Bronchial hyperresponsiveness

BHR was determined in the sixth year of life as the dose of metacholine required to induce a $20 \%$ fall in forced expiratory volume in $1 \mathrm{~s}$ (PD20), measured by spirometry [21].

Tobacco smoke exposure

Environmental tobacco smoke (ETS) exposure in utero was dichotomised by maternal smoking pattern as either having smoked during third trimester of pregnancy or not.

\section{Genotyping and single-nucleotide polymorphism analysis}

DNA was purified from blood cells from probands and parents and multiple single-nucleotide polymorphisms (SNPs) were genotyped genome wide using high throughput Illumina Infinium II HumanHap550 BeadChip technology (Illumina, Inc., San Diego, CA, USA) [22, 23].

Linkage-disequilibrium calculation and visualisation of $\mathrm{r}^{2}$ values was performed in Haploview version 4.2 (Broad Institute, Cambridge, MA, USA) [24, 25].

Quality control of genotyping included "missingness" per SNP and per individual, and Hardy-Weinberg equilibrium. Correct familial relations were double checked by Mendel error rates and identity-by-descent analyses. All quality control was performed using PLINK (Massachusetts General Hospital and the Broad Institute of Harvard \& MIT, Cambridge, MA, USA) [26, 27].

We aimed to analyse all regulatory elements by including a distance of $\pm 10 \mathrm{~kb}$ from transcription start site. The selected region was furthermore based on recombination rates from CEU population (i.e. Utah residents with Northern and Western European ancestry) made available by the International HapMap project $[28,29]$.

Allele frequencies and linkage disequilibrium pattern in the study and CEU populations were compared. Finally, we annotated the SNPs for functional effects.

\section{Statistical analysis}

SNPs were analysed for association with recurrent TLS, asthma, atopic dermatitis and BHR, respectively, performed in PLINK, in an additive genetic model by either logistic or linear regression, and in a familybased method using the transmissions disequilibrium test (TDT). In the TDT, the untransmitted alleles are set as the reference group. For further information on TDT, see the PLINK webpage [26]. The three phenotypes of recurrent TLS were analysed separately using the remaining phenotypes and/or asymptomatic children as controls.

We investigated whether $P C D H 1$ variants showed interaction with children's exposure to environmental tobacco smoke in utero, resulting in severe lung symptoms in childhood.

We used a Bonferroni corrected $\alpha$ level $(0.05 / 8=\mathrm{p}<0.00625)$ as the statistical significance threshold. Development of asthma and atopic dermatitis was visualised in Kaplan-Meier plots using R-project software [30]. Groups with one or two minor alleles were merged due to the low number of minor allele homozygous probands (asthma Kaplan-Meier: 15 homozygous probands; and atopic dermatitis KaplanMeier: 10 homozygous probands)

\section{Results}

Baseline

Study population

411 infants, aged 1 month, were included in the study. At 3 years, 345 children were still adhering to the study protocol, and 336 at 7 years.

\section{Genotyping}

Blood samples for genotyping of parents and infants were collected at the beginning of the study. After performing quality control of the genotype data (described below) and after removing one of the siblings in each sibling pair (a total of 15 probands), 362 children and 552 parents were left for analyses.

\section{Diagnoses}

Recurrent TLS was diagnosed longitudinally throughout the study. Of the initial 411 children, 139 children were categorised as having one of the three recurrent TLS phenotypes and 151 children were asymptomatic (127 of the genotyped had a recurrent TLS phenotype and 136 where asymptomatic). Asthma was also diagnosed longitudinally. By age 7 years, 70 children had, at some point during childhood, had an asthma diagnosis (66 of the genotyped children). Atopic dermatitis was also diagnosed longitudinally and by age 
7 years, 174 children had, at some point during their childhood, had atopic dermatitis (154 of the genotyped children).

Current asthma by age 7 years was determined in 47 children ( 45 of the genotyped children). BHR was measured by PD20 in 253 children (230 of the genotyped children) by age 6 years and 6 months (online supplementary data 1$)$.

\section{Genotyping}

The final region chosen for analysis (141 205 502-141248 $128 \mathrm{bp}$, build 36) included the 10-kb upstream region and $\sim 7 \mathrm{~kb}$ of the downstream region, thus excluding a peak in recombination (online supplementary data 2). The region of analysis contained eight SNPs: three positioned downstream, none positioned upstream and five positioned within transcription region (online supplementary data 3 ). The closest upstream SNP was placed $>21 \mathrm{~kb}$ away from transcription start site and was due to the distance not included in the region of interest.

Allele frequencies in the CEU-HapMap population and the study cohort were comparable (online supplementary data 6).

The linkage disequilibrium pattern in the study population revealed low correlation between all eight SNPs expressed, as $\mathrm{r}^{2}$ was between 0.00 and 0.62 (online supplementary data 4), as well as in the CEU-HapMap population (online supplementary data 5 ).

Subsequently, 15 siblings were removed in order to avoid bias in association analyses. Final analyses included 914 individuals in total, consisting of 362 probands and 552 parents, containing 234 complete trios (proband, mother and father). The call rate for all individuals in the final dataset was $\geqslant 99 \%$ and the call rate for all SNPs in the final dataset was $\geqslant 95 \%$. All analysed SNPs were in Hardy-Weinberg equilibrium $(\mathrm{p}>0.1)$.

Functional annotation of the SNPs showed that function of the variants was: synonymous (rs3797054), nonsynonymous (rs3822357), intronic (rs11167761, rs10063472 and rs6888135) and outside the gene or unknown (rs17208551, rs2974704 and rs17097812) (online supplementary data 6) [31].

\section{Genetic variations and associations with atopic disease \\ Recurrent TLS in early life}

A significant association was observed between the transient early respiratory symptoms phenotype and rs10063472 in a logistic model (transient early versus all: OR 1.91 per T allele (95\% CI 1.21-3.01), $\mathrm{p}=0.0058$; transient early versus asymptomatic: OR 2.00 per $\mathrm{T}$ allele $(95 \% \mathrm{CI} 1.23-3.25), \mathrm{p}=0.0053$ ) (table 1). Using the family-based method (transient early versus all: OR 1.93 per T allele (95\% CI 1.01-3.68), $\mathrm{p}=0.042$; transient early versus asymptomatic: OR 1.93 per T allele (95\% CI $1.01-3.68), \mathrm{p}=0.042)$. None of the other SNPs were associated with transient early phenotype and no SNP was associated with the lateonset or persistent phenotype.

\section{Asthmatic symptoms}

A Kaplan-Meier plot revealed that children with the rs10063472 $\mathrm{T}$ allele either heterozygotes or homozygotes, had an earlier onset of disease (fig. 1), consistent with the association to the transient early phenotype. Asthma at any point from birth until the age of 7 years, or a cross-section of asthma at

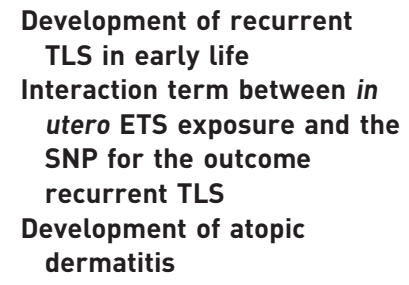

OR 2.00 per T allele

(95\% Cl 1.23-3.25), $p=0.0053^{\#}$

OR 0.46 per $T$ allele

(95\% Cl 0.13-1.68), $p=0.24$

OR 0.60 per $T$ allele (95\% Cl 0.41-0.89), $p=0.011^{\bullet}$
OR 0.73 per A allele

(95\% Cl 0.47-1.16), $p=0.18$

OR 3.67 per A allele

(95\% Cl 1.23-10.95), $p=0.020^{\circ}$

OR 0.67 per A allele (95\% Cl 0.48-0.93), $p=0.016^{\circ}$
OR 0.58 per $A$ allele

(95\% Cl 0.30-1.13), $p=0.11$

OR 0.35 per $A$ allele (95\% Cl 0.038-3.27), $p=0.36$

OR 1.86 per $A$ allele (95\% Cl 1.20-2.86), $p=0.0053^{\#}$

TLS: troublesome lung symptoms; ETS: environmental tobacco smoke. "\#: significant associations according to Bonferroni; ": nominally significant results. 


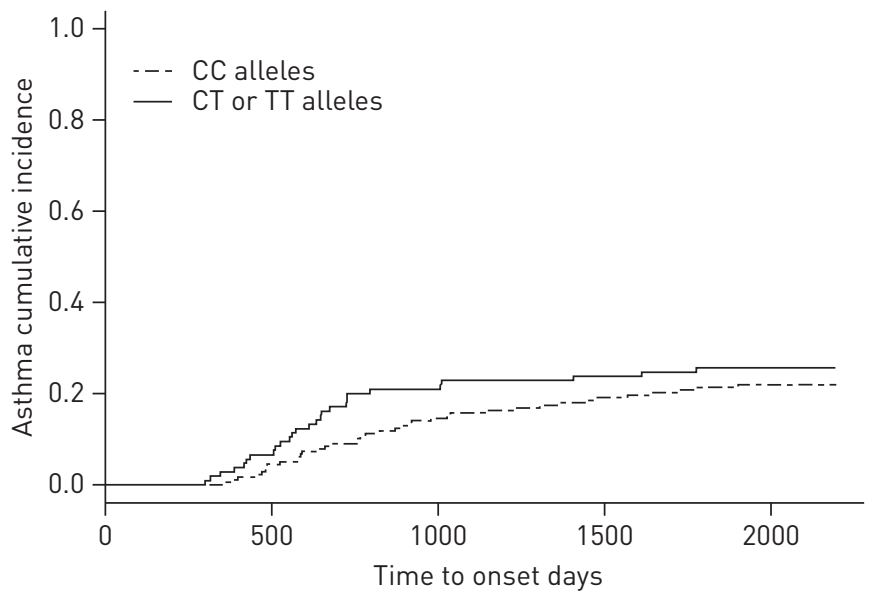

FIGURE 1 Increased risk of asthma development having the rs10063472 T allele. Kaplan-Meier plot illustrating time to onset of asthma when having one or two rs10063472 risk alleles (T allele) as opposed to having none. Due to the low number of probands being minor allele homozygous (TT), groups with one or two minor alleles were merged.

7 years, explored by logistic regression in an additive genetic model showed no statistical significance. Cox regression and/or log rank was not performed as we did not observe a constant effect over time.

\section{ETS exposure interaction}

Interaction between ETS exposure in utero and the SNPs, and development of asthmatic symptoms were tested by logistic regression with two different symptom outcomes: having asthma at any point from birth until age 7 years, and belonging to the phenotype transient early respiratory symptoms. The interaction term for having asthma was significant for rs6888135 (OR 3.56 per A allele (95\% CI 1.37-9.28), $\mathrm{p}=0.028$ ), and also for having recurrent troublesome lung symptoms in the first 3 years of life (OR 3.67 per A allele (95\% CI 1.23-10.95), $\mathrm{p}=0.020$ ) (table 1).

We furthermore repeated the association analysis on the transient early phenotype, but now stratified on exposure/no exposure to ETS in utero. We found no association between rs6888135 and transient early respiratory symptoms in any of the groups ( 15 cases and 41 controls in the exposed group, and 44 cases and 163 controls in the unexposed group).

We did rediscover the association between transient early respiratory symptoms and rs 10063472 in the unexposed group.

\section{Atopic dermatitis}

Children diagnosed with atopic dermatitis during the first 7 years of life were compared with children with no atopic dermatitis in the same period. A significant association was seen between rs11167761 and the development of atopic dermatitis, using logistic regression (OR 1.86 per A allele (95\% CI 1.20-2.86), $\mathrm{p}=0.0053$ ) (table 1). The TDT showed a comparable result but at a lower significance level (OR $1.59(95 \%$ CI 1.00-2.53), $\mathrm{p}=0.050)$. This increased risk of developing atopic dermatitis, per A allele, was visualised by Kaplan-Meier analysis, stratifying on number of effect alleles (fig. 2).

The allele increasing the risk of developing transient recurrent TLS, the rs10063472 $\mathrm{T}$ allele, showed an atopic dermatitis risk reduction (OR 0.60 per T allele (95\% CI 0.41-0.89), p=0.011). Although this was nonsignificant at the Bonferroni threshold, time to onset of atopic dermatitis, as visualised by Kaplan-Meier analysis, showed at trend towards risk reduction (fig. 3).

Furthermore, the allele that showed interaction with maternal smoking and increased development of lung symptoms in the child (rs6888135 A allele) also showed risk reduction in relation to atopic dermatitis (OR 0.67 per A allele (95\% CI 0.48-0.93), $\mathrm{p}=0.016$ ). This was not significant at the Bonferroni threshold (fig. 4). The two SNPs associated with atopic dermatitis had a very low correlation of $\mathrm{r}^{2}=0.03$ (online supplementary data 4 ).

\section{Bronchial hyperresponsiveness}

BHR, as PD20, was tested by linear regression and showed no significant association with any of the SNPs (online supplementary data 7). 
FIGURE 2 Increased risk of atopic dermatitis development having the rs11167761 A allele. Kaplan-Meier plot illustrating time to onset of atopic dermatitis when having one or two rs11167761 risk-alleles (A allele) as opposed to having none. Due to low number of probands being minor allele homozygous (AA), groups with one or two minor alleles were merged.

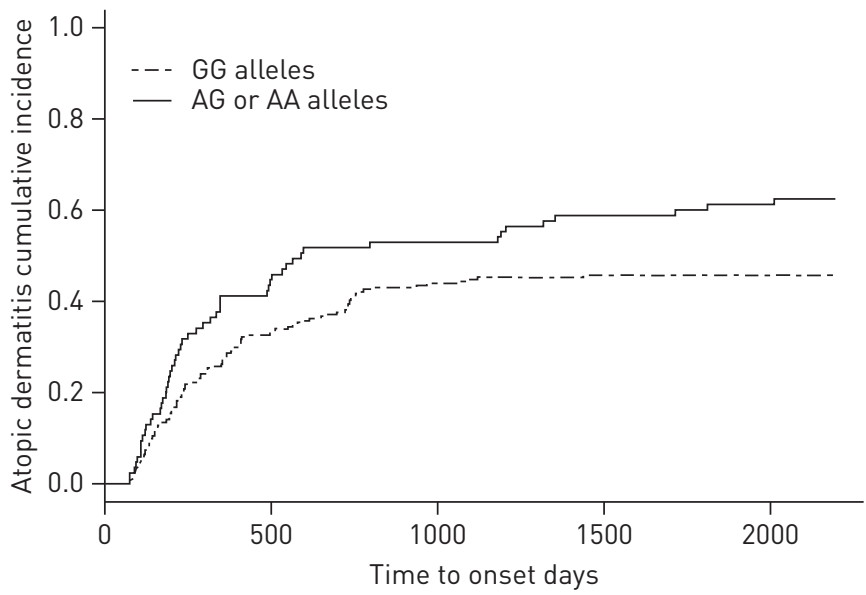

\section{Discussion}

Key findings

Common variations in $\mathrm{PCDH} 1$ increase the risk of having recurrent troublesome lung symptoms during the first 3 years of life, accelerate time to onset of asthma during that same period, and are associated with the development of atopic dermatitis.

\section{Strengths and limitations of the study}

\section{Strengths}

The COPSAC2000 study is a single-centre clinical birth cohort. Asthma, as well as atopic dermatitis, was diagnosed according to a standard operating procedure: all children were diagnosed and monitored at the clinic and not by their general practitioner, ensuring standardised diagnosis, treatment and regular evaluation.

\section{Limitations}

COPSAC2000 is a high-risk study population of asthmatic mothers and this has been accommodated, first, by comparison of allele frequencies and linkage disequilibrium, which show similarity to the general population and, secondly, by using controls from within the cohort, and thus being at the same risk of developing atopic diseases as the cases.

The genotyping, for this study, of multiple SNPs in probands and parents was performed before 2012, when the studies by Koning et al. [9] and Toncheva et al. [32] were published. We were, for that reason, unfortunately not able to analyse the polymorphisms rs7719391 and IVS3_116 in this present study.

\section{Meaning of this study}

We found an association between the SNP rs10063472 (T allele increased risk) and recurrent troublesome lung symptoms before age 3 years. Asthma onset was accelerated by the T allele of this SNP. This is a novel

FIGURE 3 Risk reduction in atopic dermatitis development having the rs10063472 T allele. Kaplan-Meier plot illustrating the risk reduction of time to onset of atopic dermatitis when having one or two rs10063472 minor-alleles (T allele) as opposed to having none. Due to the low number of probands being minor allele homozygous (TT), groups with one or two minor alleles were merged.

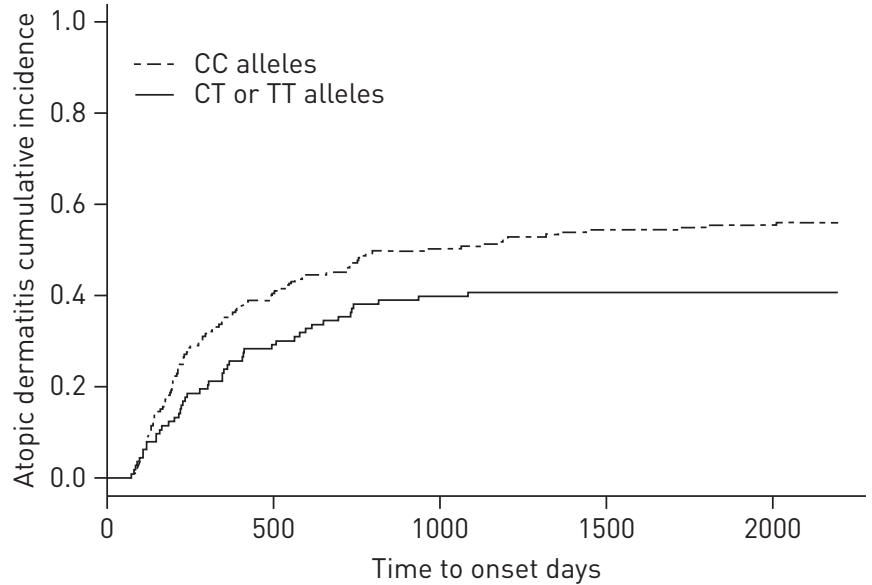




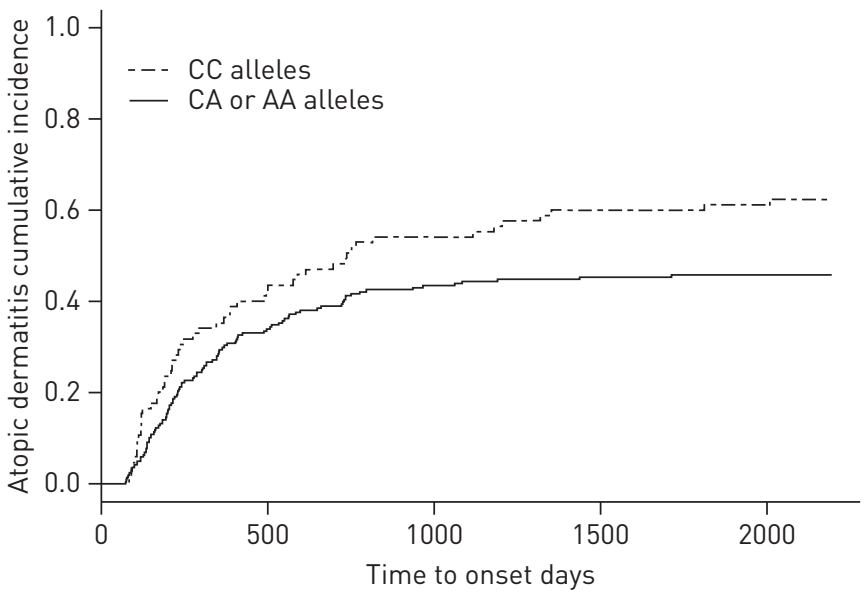

FIGURE 4 Risk reduction in atopic dermatitis development having the rs6888135 A allele. Kaplan-Meier plot illustrating the risk-reduction of time to onset of atopic dermatitis when having one or two rs6888135 minor-alleles (A allele) as opposed to having none. Due to the low number of probands being minor allele homozygous (AA), groups with one or two minor alleles were merged in visualisation. Furthermore, the rs6888135 A allele showed interaction with exposure to tobacco smoke, increasing the risk of developing recurrent troublesome lung symptoms in children (interaction not shown here).

finding not previously reported. The previously reported findings of association between asthma and the rs3797054 did not replicate in our study. This previous report, by KopPELMAN et al. [8], included one main population consisting of 200 families ascertained through probands with asthma, who were characterised between 1962 and 1975, and seven heterogeneous replication populations (population 1: Dutch trios, probands with asthma, adults with mean age 33.8 years; population 2: Caucasian general cohort, adults with mean age 51.7 years; population 3: Caucasians, Hispanic, other, general cohort, teenagers with mean age 10.7 years; population 4: UK citizens, probands with asthma, children with mean age 11.4 years; and populations 5, 6 and 7: Caucasians, Hispanics, African-Americans, case-control designs, adults with mean age $27.1,17.4$ and 24.4 years, respectively). In the main study population, asthma was diagnosed by algorithm combining symptoms, BHR and reversibility. Two paediatric cohorts were analysed by KonING et al. [9] in a subsequent report, showing no association between rs3797054 and rs3822357 and asthma ("doctor diagnosed" by any doctor or use of ICS) [9], which is in agreement with our study. In the most recent study, TonCHEVA et al. [32] found an association between a SNP not included in our genotyping, rs7719391, and a decrease in asthma in school children. Furthermore, in a subdivision of phenotypes, an association between rs2974704 and a decrease in nonatopic asthma in school children, and an association between rs11167761 and an increase in nonatopic asthma in school children were found. No SNPs were associated with atopic asthma in their study. Toncheva et al. [32] pooled two German/Austrian child populations, all asthma cases from the Multicenter Asthma Genetics in Childhood Study (MAGICS) [33] and randomly chosen children from the International Study of Asthma and Allergies in Childhood (ISAAC) II study [34]. They included 1311 children (651 asthmatics, 652 controls and eight missing status) and analysed 14 tagging SNPs. In the ISAAC II population, all children were aged 9-11 years. Asthma was assessed based on parents' reports of a physician diagnosis of asthma and/or recurrent spastic or asthmatic bronchitis and atopy was defined as a positive skin prick test. In the MAGICS population children had a mean age of 11 years (range 3-17 years), and asthma was diagnosed by a paediatric pulmonologist and atopy by a positive radioallergosorbent blood test. TonCHEVA et al. [32] did not find an association between rs 10063472 and development of asthma in school children. We were not able to replicate their findings of an association between rs11167761 and asthma subphenotypes in school children in our cohort of preschool children.

We observed a significant interaction between the rs6888135 polymorphism and in utero tobacco exposure. Our findings are only borderline significant, and previous studies on this interaction are inconsistent. In the aforementioned study, KoppeLMAN et al. [8] found an interaction between a different polymorphism (rs3822357) and ETS in utero, during the first year of life and development of BHR in childhood (age not reported) in a single high-risk family population (UK citizens, $n=340$ ), but this was not replicated in the other populations included in this study. This indicates a possible interaction between smoking and SNPs in this locus, but needs further confirmation. In our cohort, rs6888135 and rs3822357 were weakly correlated $\left(\mathrm{r}^{2}=0.03\right)$. Neither Koning et al. [9] nor TonCHEVA et al. [32] assessed interactions with ETS in utero and development of lung symptoms in the children. 
We found an association between the SNP rs11167761 and development of atopic dermatitis not previously investigated. Koning et al. [9] studied two paediatric cohorts and found an association between a 3-bp deletion (IVS3-116) and atopic dermatitis. They also investigated rs3797054 and rs3822357. The first was not found to be statistically significant, but the latter was in their high-risk study cohort $(n=967)$, but not in the general cohort $(\mathrm{n}=1560)$, and also not in their pooled analysis. ToncheVA et al. [32] assessed atopic eczema in 734 (131 cases) school children from the ISAAC II study. They found no significant association with any PCDH1 polymorphisms. Details of rs3822357 and IVS3_116 previously reported by KonING et al. [9] were, in line with our study, nonsignificant, as were indications in the present study that rs11167761 is associated with the development of atopic dermatitis in preschool children. It is noteworthy that ToNCHEVA et al. [32], however, found a significant association between rs11167761 and an increased the risk of nonatopic asthma in school children.

One unpredicted discovery we made was the risk reduction in relation to atopic dermatitis of the allele increasing the risk of asthma. We are currently not able to offer a biological explanation for this observation.

We found no association between BHR (at age 6 years) and any PCDH1-associated SNP in our study. This is in contrast with the study by KOPPELMAN et al. [8], which reported an association between rs3797054 and BHR in four populations (two high risk and two general) including adults or teenagers. However, they did not replicate this association in two family populations with asthma or three adult case-control studies. rs3822357 was associated with BHR in two cohorts, one general population of teenagers and one high-risk family cohort, but this association did not replicate in the remaining seven populations. KOPPELMAN et al. [8] also found the base pair deletion, IVS3-116, to be associated with BHR in three populations (two high risk adult populations and one case-control with adults) [8].

In agreement with our findings, KonING et al. [9] found no association between any PCDH1 polymorphism and the BHR (at age 8 years) in two paediatric cohorts (one high risk and one general). Also in agreement with our findings, ToNCHEva et al. [32] assessed BHR in 344 (71 cases) school children aged 9-11 years from the ISAAC II study and found no significant association between any genetic variants in PCDH1 and BHR. More specifically, rs3797054, rs3822357 and IVS3_116, reported by KoppeLMAN et al. [8], were found to be insignificant.

Our negative findings regarding rs3797054, rs3822357 and asthma at school age is in line with previous findings. The study by KOPPELman et al. [8] (eight populations of children and adults), the study by KonING et al. [9] (two child-only populations) and finally the study by ToNCHEvA et al. [32] (two school-age child populations) all included del IVS3-116, rs3797054 and rs3822357 in their analyses. In those studies, only inconsistent associations were seen for these variations. In particular, discrepancies between adult and child populations were apparent. Associations with any $P C D H 1$ polymorphisms and both asthma and BHR failed replication in the child cohorts by KONING et al. [9], and association with any PCDH1 polymorphisms and asthma also failed replication in the child and family cohorts by KOPPELMAN et al. [8]. Finally, associations with del IVS3-116, rs3797054 and rs3822357 and asthma and BHR failed replication by ToNCHEVA et al. [32] in their child populations.

Our novel discovery associates asthmatic symptoms to a SNP not previously reported to affect asthma development. This is the first time that asthmatic symptoms in preschool children has been associated with variation in PCDH1. Our study is the first to investigate this particular SNP in relation to the onset of asthma in children this early in life. The effect of the variation is specifically observed in children much younger than those in previous studies, which makes these results essential to the contribution of the overall understanding of $\mathrm{PCDH} 1$ and its influence on asthma development. Particularly, the thorough endotyping, as well as the early identification of asthmatic symptoms in our cohort, enabled us to distinguish the specific group of children affected by this variation.

In accordance with our novel results, as well as previously well-illustrated results, it seems apparent that common genetic polymorphisms in the $\mathrm{PCDH} 1$ gene do influence the development of asthma and atopic dermatitis. We are witnessing yet another clue as to how various underlying mechanisms lead to asthma, and this supports the relevance of subdividing it into clinically characteristic phenotypes with distinct symptom patterns, prognoses and, in the future, perhaps even treatment.

\section{Conclusions}

Common variations in $\mathrm{PCDH} 1$ increase the risk of developing both asthma and atopic dermatitis in early life. This study supports the overall hypothesis that $P C D H 1$ plays a role in the development of atopic diseases and, furthermore, offers new insight into its role in early childhood. 


\section{Acknowledgements}

We gratefully express our gratitude to the children and families of the COPSAC2000 cohort study for all their support and commitment. We acknowledge and appreciate the unique efforts of the COPSAC research team.

\section{References}

Eder W, Ege MJ, von Mutius E. The asthma epidemic. N Engl J Med 2006; 355: 2226-2235.

Kocevar VS, Bisgaard H, Jonsson L, et al. Variations in pediatric asthma hospitalization rates and costs between and within nordic countries. Chest 2004; 125: 1680-1684.

Wiesch DG, Meyers DA, Bleecker ER. Genetics of asthma. J Allergy Clin Immunol 1999; 104: 895-901.

Global Initiative for Asthma. Global Strategy for Asthma Management and Prevention. GINA, 2012. www. ginasthma.org/documents/4

5 Martinez FD, Wright AL, Taussig LM, et al. Asthma and wheezing in the first six years of life. The group health medical associates. $N$ Engl J Med 1995; 332: 133-138.

6 Henderson J, Granell R, Heron J, et al. Associations of wheezing phenotypes in the first 6 years of life with atopy, lung function and airway responsiveness in mid-childhood. Thorax 2008; 63: 974-980.

7 Moffatt MF, Gut IG, Demenais F, et al. A large-scale, consortium-based genomewide association study of asthma. N Engl J Med 2010; 363: 1211-1221.

8 Koppelman GH, Meyers DA, Howard TD, et al. Identification of PCDH1 as a novel susceptibility gene for bronchial hyperresponsiveness. Am J Respir Crit Care Med 2009; 180: 929-935.

9 Koning H, Postma DS, Brunekreef B, et al. Protocadherin-1 polymorphisms are associated with eczema in two dutch birth cohorts. Pediatr Allergy Immunol 2012; 23: 270-277.

10 Koning H, Sayers I, Stewart CE, et al. Characterization of protocadherin-1 expression in primary bronchial epithelial cells: Association with epithelial cell differentiation. FASEB J 2012; 26: 439-448.

11 Fitsialos G, Chassot AA, Turchi L, et al. Transcriptional signature of epidermal keratinocytes subjected to in vitro scratch wounding reveals selective roles for ERK1/2, p38, and phosphatidylinositol 3-kinase signaling pathways. J Biol Chem 2007; 282: 15090-15102.

12 Redies C, Vanhalst K, Roy F. Delta-protocadherins: unique structures and functions. Cell Mol Life Sci 2005; 62: 2840-2852.

13 Vanhalst K, Kools P, Staes K, et al. Delta-protocadherins: a gene family expressed differentially in the mouse brain. Cell Mol Life Sci 2005; 62: 1247-1259.

14 Wu Q, Maniatis T. A striking organization of a large family of human neural cadherin-like cell adhesion genes. Cell 1999; 97: 779-790.

15 Redies C, Heyder J, Kohoutek T, et al. Expression of protocadherin-1 (PCDH1) during mouse development. Dev Dyn 2008; 237: 2496-2505.

16 GenBank N. www.ncbi.nlm.nih.gov/gene/5097 Date last accessed: September 27, 2013.

17 Bonnelykke K, Pipper CB, Tavendale R, et al. Filaggrin gene variants and atopic diseases in early childhood assessed longitudinally from birth. Pediatr Allergy Immunol 2010; 21: 954-961.

18 Bisgaard H. The Copenhagen Prospective Study on Asthma in Childhood (COPSAC): design, rationale, and baseline data from a longitudinal birth cohort study. Ann Allergy Asthma Immunol 2004; 93: 381-389.

19 Bisgaard H, Pipper CB, Bonnelykke K. Endotyping early childhood asthma by quantitative symptom assessment. J Allergy Clin Immunol 2011; 127: 1155-1164.

20 Halkjaer LB, Loland L, Buchvald FF, et al. Development of atopic dermatitis during the first 3 years of life: The copenhagen prospective study on asthma in childhood cohort study in high-risk children. Arch Dermatol 2006; 142: 561-566.

21 Bisgaard H, Pedersen S, Anhoj J, et al. Determinants of lung function and airway hyperresponsiveness in asthmatic children. Respir Med 2007; 101: 1477-1482.

22 Sleiman PM, Flory J, Imielinski M, et al. Variants of DENND1B associated with asthma in children. N Engl J Med 2010; 362: 36-44.

23 Hakonarson H, Grant SF, Bradfield JP, et al. A genome-wide association study identifies KIAA0350 as a type 1 diabetes gene. Nature 2007; 448: 591-594.

24 Barrett JC, Fry B, Maller J, et al. Haploview: analysis and visualization of ld and haplotype maps. Bioinformatics 2005; 21: 263-265.

25 Broad Institute. Haploview. www.broadinstitute.org/haploview/haploview Date last accessed: September 27, 2013. PLINK. http://pngu.mgh.harvard.edu/ purcell/plink/ Date last accessed: September 27, 2013.

Purcell S, Neale B, Todd-Brown K, et al. PLINK: a tool set for whole-genome association and population-based linkage analyses. Am J Hum Genet 2007; 81: 559-575.

28 HapMap. International HapMap project. http://hapmap.ncbi.nlm.nih.gov/ Date last accessed: September 27, 2013. International HapMap Consortium. The International HapMap Project. Nature 2003; 426: 789-796.

30 R-project development core team. R-project: a language and environment for statistical computing. www.r-project. org/ Date last accessed: September 27, 2013.

31 Abecasis Lab Gds. www.sph.umich.edu/csg/abecasis/MACH/download/1000G-PhaseI-Interim.html Date last accessed: May 6, 2013.

32 Toncheva AA, Suttner K, Michel S, et al. Genetic variants in protocadherin-1, bronchial hyper-responsiveness, and asthma subphenotypes in german children. Pediatr Allergy Immunol 2012; 23: 636-641.

33 Michel S, Liang L, Depner M, et al. Unifying candidate gene and GWAS approaches in asthma. PLoS One 2010; 5: e13894.

34 Moffatt MF, Kabesch M, Liang L, et al. Genetic variants regulating ORMDL3 expression contribute to the risk of childhood asthma. Nature 2007; 448: 470-473. 